\title{
Control of the non-stationary spin-polarized tunneling currents by applied bias changing
}

\author{
N.S. Maslova ${ }^{1}$, P.I. Arseyev ${ }^{2}$, and V.N. Mantsevich ${ }^{\text {I }}$ \\ ${ }^{1}$ Moscow State University, 119991 Moscow, Russia, \\ 2 P.N. Lebedev Physical Institute RAS, 119991 Moscow, Russia
}

(Dated: November 10, 2018)

\begin{abstract}
We reveal that for the single Anderson impurity localized between non-magnetic leads of the tunneling contact "magnetic" state can be distinguished from the "paramagnetic" one only by the analysis of the non-stationary system characteristics or the behavior of the second order correlation functions for the localized electrons occupation numbers. We investigate the response of the system to the sudden shift of the applied bias and to the switching "on" the coupling to the second lead of the tunneling contact. We demonstrate that in addition to the changes of the relaxation regimes and typical relaxation time scales, non-stationary spin-polarized currents flowing in the both leads are present in the system. Spin polarization and direction of the non-stationary currents in each lead can be simultaneously inverted by the sudden changing of the applied bias voltage.
\end{abstract}

PACS numbers: 72.25.-b, 72.15.Lh, 73.63.-b

\section{INTRODUCTION}

Physics of spin-polarized electron transport in semiconductor nanostructures is among the most rapidly developing topics now a days 1 . Significant progress has been achieved in experimental and theoretical investigation of spin-polarized stationary transport in magnetic tunneling junctions ${ }^{2}, 3,4,5,6$. Different magnetic materials, such as ferromagnetic metals 7 or diluted magnetic semiconductors ${ }^{8}$ have been applied as a spin injection sources and drains. Nevertheless spin-polarized current sources, that use nonmagnetic materials are attractable as they enable to avoid the presence of accidental magnetic field that may cause undesirable effects on the spin currents. It was demonstrated recently, that electron tunneling could be spin dependent even in the case of nonmagnetic leads, 10. Moreover, spin-filter devices, which can generate a spin-polarized current without using magnetic properties of materials were proposed in 11,12 .

To the best of our knowledge stationary spin-polarized currents are usually under investigation. However, creation, diagnostics and controlled manipulation of charge and spin states of the impurity atoms or quantum dots (QDs), applicable for ultra small size electronic devices design requires careful analysis of non-stationary effects and transient processes $13,14,15,16,17,18$. Consequently, time dependent dynamics of initial spin and charge configurations of correlated impurity or QD is an area of great interest both from fundamental and technological point of view. Moreover, characteristics of the stationary state of single impurity interacting with the reservoir in the presence of strong Coulomb correlations are not completely understood ${ }^{19}, 20,21,22,23$.

In this paper we analyze non-stationary spin polarized currents through the single-impurity state localized in the tunnel junction in the presence of Coulomb correlations and applied bias voltage. We demonstrate that "magnetic" state can be distinguished from the "paramagnetic" one only by analyzing time evolution of opposite spin electron occupation numbers. We reveal that non-stationary spin-polarized currents can flow in the both leads and their direction and polarization depend on the value of applied bias. Moreover, nonstationary spin-polarized currents simultaneously change their polarization and sign with the applied bias voltage variations.

\section{THEORETICAL MODEL}

We consider non-stationary processes in the system of single-level impurity placed between two non-magnetic electronic reservoirs (tunneling junction) with Coulomb correlations of localized electrons. The Hamiltonian of the system

$$
\hat{H}=\hat{H}_{i m p}+\hat{H}_{r e s}+\hat{H}_{t u n}
$$

is written as a sum of the single-level Anderson impurity Hamiltonian

$$
\hat{H}_{i m p}=\sum_{\sigma} \varepsilon_{1} \hat{n}_{1 \sigma}++U \hat{n}_{1 \sigma} \hat{n}_{1-\sigma},
$$

non-magnetic electronic reservoirs Hamiltonian

$$
\hat{H}_{r e s}=\sum_{k \sigma} \varepsilon_{k} \hat{c}_{k \sigma}^{+} \hat{c}_{k \sigma}+\sum_{p \sigma}\left(\varepsilon_{p}-e V\right) \hat{c}_{p \sigma}^{+} \hat{c}_{p \sigma}
$$

and the tunneling part

$$
\hat{H}_{t u n}=\sum_{k \sigma} t_{k}\left(\hat{c}_{k \sigma}^{+} \hat{c}_{1 \sigma}+\hat{c}_{1 \sigma}^{+} \hat{c}_{k \sigma}\right)+\sum_{p \sigma} t_{p}\left(\hat{c}_{p \sigma}^{+} \hat{c}_{1 \sigma}+\hat{c}_{1 \sigma}^{+} \hat{c}_{p \sigma}\right)
$$


Here index $k(p)$ labels continuous spectrum states in the leads, $t_{k(p)}$ is the tunneling transfer amplitude between continuous spectrum states and localized state with the energy $\varepsilon_{1}$ which is considered to be independent on the momentum and spin. Operators $\hat{c}_{k(p)}^{+} / \hat{c}_{k(p)}$ are the creation/annihilation operators for the electrons in the continuous spectrum states $k(p) . \quad \hat{n}_{1 \sigma(-\sigma)}=$ $\hat{c}_{1 \sigma(-\sigma)}^{+} \hat{c}_{1 \sigma(-\sigma)}$-localized state electron occupation numbers, where operator $\hat{c}_{1 \sigma(-\sigma)}$ destroys electron with the spin $\sigma(-\sigma)$ on the energy level $\varepsilon_{1}$. $U$ is the on-site Coulomb repulsion for the double occupation of the localized state. Our analysis deals with the low temperature regime when the Fermi level is well defined and the temperature is much lower than all the typical energy scales in the system. Consequently the distribution function of the electrons in the leads (band electrons) is close to the Fermi step.

\section{NON-STATIONARY ELECTRONIC TRANSPORT: FORMALISM AND RESULTS}

Let us further consider $\hbar=1$ and $e=1$ elsewhere, so the motion equations for the electron operators products $\hat{c}_{1 \sigma}^{+} \hat{c}_{1 \sigma}, \hat{c}_{1 \sigma}^{+} \hat{c}_{k(p) \sigma}$ and $\hat{c}_{k^{\prime}(p) \sigma}^{+} \hat{c}_{k(p) \sigma}$ can be written as:

$$
\begin{aligned}
i \frac{\partial \hat{c}_{1 \sigma}^{+} \hat{c}_{1 \sigma}}{\partial t}= & -\sum_{k, \sigma} t_{k}\left(\hat{c}_{k \sigma}^{+} \hat{c}_{1 \sigma}-\hat{c}_{1 \sigma}^{+} \hat{c}_{k \sigma}\right)- \\
& -\sum_{p, \sigma} t_{p}\left(\hat{c}_{p \sigma}^{+} \hat{c}_{1 \sigma}-\hat{c}_{1 \sigma}^{+} \hat{c}_{p \sigma}\right)
\end{aligned}
$$

$$
\begin{aligned}
i \frac{\partial \hat{c}_{1 \sigma}^{+} \hat{c}_{k \sigma}}{\partial t}= & -\left(\varepsilon_{1}-\varepsilon_{k}\right) \hat{c}_{1 \sigma}^{+} \hat{c}_{k \sigma}- \\
& -U \hat{n}_{1-\sigma} \hat{c}_{1 \sigma}^{+} \hat{c}_{k \sigma}+t_{k}\left(\hat{n}_{1 \sigma}-\hat{n}_{k \sigma}\right)- \\
& -\sum_{k^{\prime} \neq k} t_{k^{\prime}} \hat{c}_{k^{\prime} \sigma}^{+} \hat{c}_{k \sigma}-\sum_{p} t_{p} \hat{c}_{p \sigma}^{+} \hat{c}_{k \sigma},
\end{aligned}
$$

$$
\begin{aligned}
i \frac{\partial \hat{c}_{k^{\prime} \sigma}^{+} \hat{c}_{k \sigma}}{\partial t}= & -\left(\varepsilon_{k^{\prime}}-\varepsilon_{k}\right) \cdot \hat{c}_{k^{\prime} \sigma}^{+} \hat{c}_{k \sigma}- \\
& -t_{k^{\prime}} \hat{c}_{1 \sigma}^{+} \hat{c}_{k \sigma}+t_{k} \hat{c}_{k^{\prime} \sigma}^{+} \hat{c}_{1 \sigma}
\end{aligned}
$$

and

$$
\begin{aligned}
i \frac{\partial \hat{c}_{p \sigma}^{+} \hat{c}_{k \sigma}}{\partial t}= & \left(\varepsilon_{k}-\varepsilon_{p}\right) \cdot \hat{c}_{p \sigma}^{+} \hat{c}_{k \sigma}+ \\
& +t_{k} \cdot \hat{c}_{p \sigma}^{+} \hat{c}_{1 \sigma}-t_{p} \cdot \hat{c}_{1 \sigma}^{+} \hat{c}_{k \sigma}
\end{aligned}
$$

where $\hat{n}_{k}=\hat{c}_{k \sigma}^{+} \hat{c}_{k \sigma}$ is an occupation operator for the electrons in the reservoir.

Equations of motion for the electron operators products $\hat{c}_{1 \sigma}^{+} \hat{c}_{p \sigma}$ and $\hat{c}_{p^{\prime} \sigma}^{+} \hat{c}_{p \sigma}$ can be obtained from Eq. 6 . and Eq. (7) correspondingly by the indexes substitution $k \leftrightarrow p$ and $k^{\prime} \leftrightarrow p^{\prime}$.

One can easily obtain:

$$
\begin{aligned}
\sum_{k^{\prime} \neq k} \hat{c}_{k^{\prime} \sigma}^{+} \hat{c}_{k \sigma} t_{k^{\prime}} & =i \sum_{k^{\prime}} \int^{t} d t_{1} e^{i \cdot\left(\varepsilon_{k}-\varepsilon_{k}^{\prime}\right) \cdot\left(t-t_{1}\right)} \times \\
& \times\left(t_{k^{\prime}}^{2} \cdot \hat{c}_{1 \sigma}^{+} \hat{c}_{k \sigma}-t_{k} t_{k^{\prime}} \cdot \hat{c}_{k^{\prime} \sigma}^{+} \hat{c}_{1 \sigma}\right) .
\end{aligned}
$$

Similar expressions for the products $\hat{c}_{p^{\prime} \sigma}^{+} \hat{c}_{p \sigma}, \hat{c}_{p^{\prime} \sigma}^{+} \hat{c}_{k \sigma}$ and so on can be obtained from expression (9) by the indexes changing $k \leftrightarrow p, k^{\prime} \leftrightarrow p^{\prime}$ and so on.

Substituting (9) in Eq. (6) we get

$$
\begin{aligned}
i \frac{\partial \hat{c}_{1 \sigma}^{+} \hat{c}_{k \sigma}}{\partial t} & +\left(\varepsilon_{1}-\varepsilon_{k}+i \Gamma\right) \hat{c}_{1 \sigma}^{+} \hat{c}_{k \sigma}+ \\
& +U \hat{n}_{1-\sigma} \hat{c}_{1 \sigma}^{+} \hat{c}_{k \sigma}=t_{k}\left(\hat{n}_{1 \sigma}-\hat{n}_{k \sigma}\right)+ \\
& +i \sum_{k^{\prime}} \int^{t} d t_{1} t_{k} t_{k^{\prime}} \hat{c}_{k^{\prime} \sigma}^{+}\left(t_{1}\right) \hat{c}_{1 \sigma}\left(t_{1}\right) e^{i \cdot\left(\varepsilon_{k}-\varepsilon_{k}^{\prime}\right) \cdot\left(t-t_{1}\right)}+ \\
& +i \sum_{p} \int^{t} d t_{1} t_{k} t_{p} \hat{c}_{p \sigma}^{+}\left(t_{1}\right) \hat{c}_{1 \sigma}\left(t_{1}\right) e^{i \cdot\left(\varepsilon_{k}-\varepsilon_{p}\right) \cdot\left(t-t_{1}\right)},
\end{aligned}
$$

where $\Gamma=\Gamma_{k}+\Gamma_{p}$ and $\Gamma_{k(p)}=\nu_{k(p) 0} t_{k(p)}^{2}, \nu_{k(p) 0}$ - are the unperturbed densities of states in the left and right leads of the tunneling contact.

Equation for $\frac{\partial \hat{c}_{1 \sigma}^{+} \hat{c}_{p \sigma}}{\partial t}$ can be obtained from Eq. 10 by the indexes $k \leftrightarrow p$ and $k^{\prime} \leftrightarrow p^{\prime}$ changing. Multiplying Eq. 10 by the electron operators $1-\hat{n}_{1-\sigma}$ and $\hat{n}_{1-\sigma}$ we obtain the following expressions:

$$
\begin{aligned}
\left(1-\hat{n}_{1-\sigma}\right) & \cdot i \frac{\partial \hat{c}_{1 \sigma}^{+} \hat{c}_{k \sigma}}{\partial t}+\left(\varepsilon_{1}-\varepsilon_{k}+i \Gamma\right)\left(1-\hat{n}_{1-\sigma}\right) \hat{c}_{1 \sigma}^{+} \hat{c}_{k \sigma}= \\
= & \left(1-\hat{n}_{1-\sigma}\right) \cdot\left[t_{k} \cdot\left(\hat{n}_{1 \sigma}-\hat{n}_{k \sigma}\right)+\right. \\
& +i \sum_{k^{\prime}} \int^{t} d t_{1} t_{k} t_{k^{\prime}} \hat{c}_{k^{\prime} \sigma}^{+}\left(t_{1}\right) \hat{c}_{1 \sigma}\left(t_{1}\right) e^{i \cdot\left(\varepsilon_{k}-\varepsilon_{k}^{\prime}\right) \cdot\left(t-t_{1}\right)}+ \\
& \left.+i \sum_{p} \int^{t} d t_{1} t_{k} t_{p} \hat{c}_{p \sigma}^{+}\left(t_{1}\right) \hat{c}_{1 \sigma}\left(t_{1}\right) e^{i \cdot\left(\varepsilon_{k}-\varepsilon_{p}\right) \cdot\left(t-t_{1}\right)}\right]
\end{aligned}
$$

and 


$$
\begin{aligned}
\hat{n}_{1-\sigma} & \cdot i \frac{\partial \hat{c}_{1 \sigma}^{+} \hat{c}_{k \sigma}}{\partial t}+\left(\varepsilon_{1}-\varepsilon_{k}+U+i \Gamma\right) \hat{n}_{1-\sigma} \hat{c}_{1 \sigma}^{+} \hat{c}_{k \sigma}= \\
& =\hat{n}_{1-\sigma} \cdot\left[t_{k} \cdot\left(\hat{n}_{1 \sigma}-\hat{n}_{k \sigma}\right)+\right. \\
& +i \sum_{k^{\prime}} \int^{t} d t_{1} t_{k} t_{k^{\prime}} \hat{c}_{k^{\prime} \sigma}^{+}\left(t_{1}\right) \hat{c}_{1 \sigma}\left(t_{1}\right) e^{i \cdot\left(\varepsilon_{k}-\varepsilon_{k}^{\prime}\right) \cdot\left(t-t_{1}\right)}+ \\
& \left.+i \sum_{p} \int^{t} d t_{1} t_{k} t_{p} \hat{c}_{p \sigma}^{+}\left(t_{1}\right) \hat{c}_{1 \sigma}\left(t_{1}\right) e^{i \cdot\left(\varepsilon_{k}-\varepsilon_{p}\right) \cdot\left(t-t_{1}\right)}\right] .
\end{aligned}
$$

If condition $\frac{\varepsilon_{1}-\varepsilon_{F}}{\Gamma}>1$ is fulfilled, $\hat{n}_{1-\sigma}$ is a slowly varying quantity in comparison with the $\hat{c}_{1 \sigma}^{+} \hat{c}_{k(p) \sigma}$ $\left(\frac{\partial}{\partial t} \hat{n}_{1-\sigma}<<\frac{\partial}{\partial t} \hat{c}_{1 \sigma}^{+} \hat{c}_{k(p) \sigma}\right)$. Consequently, it is reasonable to consider that:

$$
\frac{\partial}{\partial t} \hat{n}_{1-\sigma} \hat{c}_{1 \sigma}^{+} \hat{c}_{k(p) \sigma} \sim \hat{n}_{1-\sigma} \frac{\partial}{\partial t} \hat{c}_{1 \sigma}^{+} \hat{c}_{k(p) \sigma} .
$$

So, the terms $\left(\frac{\partial}{\partial t} \hat{n}_{1-\sigma}\right) \hat{c}_{1 \sigma}^{+} \hat{c}_{k(p) \sigma}$ are omitted. One can get expressions for $\left(1-\widehat{n}_{1-\sigma}\right) \hat{c}_{1 \sigma}^{+} \hat{c}_{k(p) \sigma}$ and $\widehat{n}_{1-\sigma} \hat{c}_{1 \sigma}^{+} \hat{c}_{k(p) \sigma}$ [using the procedure similar to the one which was used to obtain Eq. $\left[9\right.$ from Eq. (7)] and then for the $\hat{c}_{1 \sigma}^{+} \hat{c}_{k(p) \sigma}$.

Substituting expressions for the $\hat{c}_{1 \sigma}^{+} \hat{c}_{k(p) \sigma}$ and $\hat{c}_{k(p) \sigma}^{+} \hat{c}_{1 \sigma}$ to Eq. (5) we obtain equations, which determine time evolution of the electron occupation numbers $\hat{n}_{1 \sigma}$. It is necessary to note, that the last term in Eq. (10) after summation over the index $k(p)$ doesn't contribute to the non-stationary equations for the electron occupation numbers $\hat{n}_{1 \sigma}$. So, time evolution of the electron occupation numbers operators in the situation when the second lead and the non-zero bias voltage are present can be analyzed by means of the system of equations:

$$
\begin{aligned}
\frac{\hat{n}_{1 \sigma}}{\partial t} & =-2 \Gamma\left[\hat{n}_{1 \sigma}-\left(1-\hat{n}_{1-\sigma}\right) \hat{N}_{\varepsilon}^{T}(t)-\hat{n}_{1-\sigma} \hat{N}_{\varepsilon+U}^{T}(t)\right], \\
\frac{\hat{n}_{1-\sigma}}{\partial t} & =-2 \Gamma\left[\hat{n}_{1-\sigma}-\left(1-\hat{n}_{1 \sigma}\right) \hat{N}_{\varepsilon}^{T}(t)-\hat{n}_{1 \sigma} \hat{N}_{\varepsilon+U}^{T}(t)\right],
\end{aligned}
$$

where

$$
\begin{aligned}
\hat{N}_{\varepsilon}^{T}(t) & =\frac{\Gamma_{k}}{\Gamma} \cdot \hat{N}_{k \varepsilon}(t)+\frac{\Gamma_{p}}{\Gamma} \cdot \hat{N}_{p \varepsilon}(t), \\
\hat{N}_{\varepsilon+U}^{T}(t) & =\frac{\Gamma_{k}}{\Gamma} \cdot \hat{N}_{k \varepsilon+U}(t)+\frac{\Gamma_{p}}{\Gamma} \cdot \hat{N}_{p \varepsilon+U}(t)
\end{aligned}
$$

and

$$
\begin{array}{r}
\hat{N}_{k(p) \varepsilon}=\frac{1}{2} i \cdot \int d \varepsilon_{k(p)} f_{k(p)}^{\sigma}\left(\varepsilon_{k(p)}\right) \times \\
\times\left[\frac{1-e^{i\left(\varepsilon_{1}+\tilde{\Gamma}-\varepsilon_{k(p)}\right) t}}{\varepsilon_{1}+i \widetilde{\Gamma}-\varepsilon_{k(p)}}-\frac{1-e^{-i\left(\varepsilon_{1}-i \widetilde{\Gamma}-\varepsilon_{k(p)}\right) t}}{\varepsilon_{1}-i \widetilde{\Gamma}-\varepsilon_{k(p)}}\right], \\
\hat{N}_{k(p) \varepsilon+U}(t)=\frac{1}{2} i \cdot \int d \varepsilon_{k(p)} f_{k(p)}^{\sigma}\left(\varepsilon_{k(p)}\right) \times \\
\times\left[\frac{1-e^{i\left(\varepsilon_{1}+U+i \widetilde{\Gamma}-\varepsilon_{k(p)}\right) t}}{\varepsilon_{1}+U+i \widetilde{\Gamma}-\varepsilon_{k(p)}}-\frac{1-e^{-i\left(\varepsilon_{1}+U-i \widetilde{\Gamma}-\varepsilon_{k(p)}\right) t}}{\varepsilon_{1}+U-i \widetilde{\Gamma}-\varepsilon_{k(p)}}\right] .
\end{array}
$$

In Eq. (16) parameter $\Gamma$ is replaced by the effective parameter $\Gamma \simeq \Gamma$, because after applying approximation (13) to Eq. (12) the omitted terms are of the order of $\Gamma$. We can obtain equations for the occupation numbers of localized electrons $n_{1 \pm \sigma}$ by averaging Eqs. (14)-(16) for the operators and by decoupling electrons occupation numbers in the reservoir. Such decoupling procedure is reasonable if one considers that electrons in the macroscopic reservoir are in the thermal equilibrium. After decoupling one has to replace electron occupation numbers operators in the reservoir $\hat{n}_{k}^{\sigma}$ in Eqs. 115- 16 by the Fermi distribution functions $f_{k}^{\sigma}$.

If one is interested in the situation when the second lead and non-zero bias voltage are switched "on" at the time moment $t=t_{0}>0$, Eqs. (14) can be easily generalized:

$$
\begin{array}{r}
\frac{\partial n_{1 \sigma}}{\partial t}=-2 \Theta\left(t_{0}-t\right) \Gamma \times \\
\times\left[n_{1 \sigma}-\left(1-n_{1-\sigma}\right) N_{k \varepsilon}(t)-n_{1-\sigma} N_{k \varepsilon+U}(t)\right]- \\
-2 \Theta\left(t-t_{0}\right) \Gamma \times \\
\times\left[n_{1 \sigma}-\left(1-n_{1-\sigma}\right) N_{k \varepsilon}^{T}(t)-n_{1-\sigma} N_{k \varepsilon+U}^{T}(t)\right], \\
\frac{\partial n_{1-\sigma}}{\partial t}=-2 \Theta\left(t_{0}-t\right) \Gamma \times \\
\times\left[n_{1-\sigma}-\left(1-n_{1 \sigma}\right) N_{k \varepsilon}(t)-n_{1 \sigma} N_{k \varepsilon+U}(t)\right]- \\
-2 \Theta\left(t-t_{0}\right) \Gamma \times \\
\times\left[n_{1-\sigma}-\left(1-n_{1 \sigma}\right) N_{k \varepsilon}^{T}(t)-n_{1 \sigma} N_{k \varepsilon+U}^{T}(t)\right],
\end{array}
$$

where $\Gamma=\Gamma_{k}$ for $t<t_{0}$ and $\Gamma=\Gamma_{k}+\Gamma_{p}$ for $t>t_{0}$. Solution can be easily obtained by the numerical simulations of the system of equations. Time dependent dynamics of the electron occupation numbers and their correlation functions can be analyzed for the different initial conditions: 1) the non-zero localized magnetic moment exists on the impurity $\left(\left|n_{1 \sigma}-n_{1-\sigma}\right| \sim 1\right)$. Such state can be prepared due to the applied external magnetic field $\mu B>>\varepsilon_{1}$, which is switched "off" at the initial time moment $t=0 ; 2)$ the initial state close to the highly occupied paramagnetic one $\left(\left|1-n_{1 \pm \sigma}\right|<<1\right)$ can be prepared by the applied bias voltage $|e V|>\varepsilon_{1}+U$ switching "off" or "on" at the initial time moment $t=0 ; 3$ ) 
the initial state close to the low occupied paramagnetic one $\left(\left|n_{1 \pm \sigma}\right|<<1\right)$ can be prepared by the applied bias voltage $|e V|<\varepsilon_{1}$ switching "off" or "on" at the initial time moment $t=0$. It will be shown that relaxation time scale strongly depends on the properties of the initially prepared state.

If one is interested in system time evolution for the time scales $t>>\frac{1}{\varepsilon_{1}}$, fast oscillating terms, which contain time dependent exponents can be neglected and functions $N_{k(p) \varepsilon}, N_{k(p) \varepsilon+U}$ [see Eq.116]] become independent from $t$. So, the localized electrons occupation numbers $n_{1 \sigma}(t)$, $n_{1-\sigma}(t)$ can be easily found for the arbitrary initial conditions:

For $0<t<t_{0}$

$$
\begin{array}{r}
n_{1 \sigma}(t)=\frac{N_{k \varepsilon}}{1+\Delta N} \cdot\left(1-e^{\lambda_{2} t}\right)+ \\
+\frac{n_{1 \sigma}(0)-n_{1-\sigma}(0)}{2} \cdot e^{\lambda_{1} t}+\frac{n_{1 \sigma}(0)+n_{1-\sigma}(0)}{2} \cdot e^{\lambda_{2} t} \\
n_{1-\sigma}(t)=\frac{N_{k \varepsilon}}{1+\Delta N} \cdot\left(1-e^{\lambda_{2} t}\right)+ \\
+\frac{n_{1-\sigma}(0)-n_{1 \sigma}(0)}{2} \cdot e^{\lambda_{1} t}+\frac{n_{1-\sigma}(0)+n_{1 \sigma}(0)}{2} \cdot e^{\lambda_{2} t},
\end{array}
$$

where $n_{1 \pm \sigma}(0)$ are the initial conditions. For $t>t_{0}$

$$
\begin{aligned}
n_{1 \sigma}(t) & =\frac{N_{\varepsilon}^{T}}{1+\Delta N^{T}} \cdot\left(1-e^{\lambda_{2}^{T}\left(t-t_{0}\right)}\right)+ \\
& +\frac{n_{1 \sigma}\left(t_{0}\right)-n_{1-\sigma}\left(t_{0}\right)}{2} \cdot e^{\lambda_{1}^{T}\left(t-t_{0}\right)}+ \\
& +\frac{n_{1 \sigma}\left(t_{0}\right)+n_{1-\sigma}\left(t_{0}\right)}{2} \cdot e^{\lambda_{2}^{T}\left(t-t_{0}\right)}, \\
n_{1-\sigma}(t) & =\frac{N_{k \varepsilon}^{T}}{1+\Delta N^{T}} \cdot\left(1-e^{\lambda_{2}^{T}\left(t-t_{0}\right)}\right)+ \\
& +\frac{n_{1-\sigma}\left(t_{0}\right)-n_{1 \sigma}\left(t_{0}\right)}{2} \cdot e^{\lambda_{1}^{T}\left(t-t_{0}\right)}+ \\
& +\frac{n_{1-\sigma}\left(t_{0}\right)+n_{1 \sigma}\left(t_{0}\right)}{2} \cdot e^{\lambda_{2}^{T}\left(t-t_{0}\right)} .
\end{aligned}
$$

Eigenvalues $\lambda_{1,2}$ and $\lambda_{1,2}^{T}$ have the following form:

$$
\begin{aligned}
& \lambda_{1,2}=-2 \Gamma \cdot(1 \mp \Delta N), \\
& \lambda_{1,2}^{T}=-2 \Gamma \cdot\left(1 \mp \Delta N^{T}\right)
\end{aligned}
$$

and

$$
\begin{aligned}
\Delta N & =N_{\varepsilon}-N_{\varepsilon+U}, \\
\Delta N^{T} & =N_{\varepsilon}^{T}-N_{\varepsilon+U}^{T} .
\end{aligned}
$$

$n_{1 \pm \sigma}\left(t_{0}\right)$ is determined by expressions 18 for $t=t_{0}$. Relaxation rates behavior as a function of applied bias

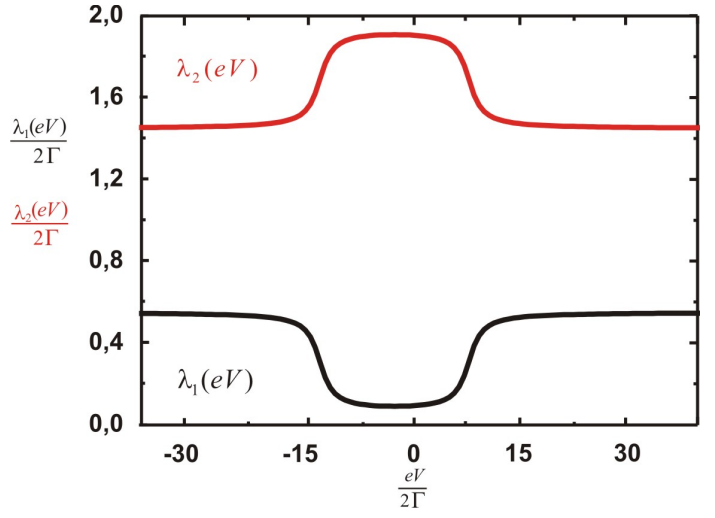

FIG. 1. (Color online) Normalized relaxation rates behavior as a functions of the applied bias voltage for the parameters $\varepsilon_{1} / 2 \Gamma=-2.5 U / 2 \Gamma=7.5$ and $\Gamma=\Gamma_{k}+\Gamma_{p}=1\left(\Gamma_{k}=\Gamma_{p}\right)$.

voltage is shown in the Fig 1 . For small values of applied bias $e V<\varepsilon$ two time scales $\left|\lambda_{1,2}^{T}\right|^{-1}$ strongly differ, while for the large absolute values of the applied bias $\frac{\left|\lambda_{1}^{T}\right|}{\left|\lambda_{2}^{T}\right|} \rightarrow$ $1 / 3$.

For the infinitely large times $t \rightarrow \infty$ the stationary state is always "paramagnetic" one and electron occupation numbers are:

$$
n_{1 \sigma}^{s t T}=n_{1-\sigma}^{s t T}=\frac{N_{\varepsilon}^{T}}{1+\Delta N^{T}}
$$

The behavior of localized state electron occupation numbers for the different initial conditions and the set of system parameters in the case, when the second lead is switched on at the time moment $t=t_{0}>0$ is depicted in the Fig. (2). Obtained results demonstrate, that switching "on" of the second lead with the non-zero applied bias results in the increasing of the relaxation rate and consequently destroys the long-living "magnetic" moment.

\section{NON-STATIONARY SPIN-POLARIZED CURRENTS: FORMALISM AND RESULTS}

If the initial state is a "magnetic" one, non-stationary spin-polarized currents $I_{k(p)}(t)^{ \pm}$flow in the each lead:

$I_{k}^{ \pm}(t)=-2 \Gamma_{k}\left[n_{1 \pm \sigma}-\left(1-n_{1 \mp \sigma}\right) N_{k \varepsilon}(t)-n_{1 \mp \sigma} N_{k \varepsilon+U}(t)\right]$
$I_{p}^{ \pm}(t)=-2 \Gamma_{p}\left[n_{1 \pm \sigma}-\left(1-n_{1 \mp \sigma}\right) N_{p \varepsilon}(t)-n_{1 \mp \sigma} N_{p \varepsilon+U}(t)\right]$,

where electron occupation numbers $n_{1 \pm \sigma}$ are determined from the system of equations $\sqrt{14}$ with the magnetic initial conditions.

Non-stationary spin-polarized currents can flow in the both leads and their direction and polarization depend on the value of applied bias. Non-stationary spin-polarized currents $I_{k(p)}(t)^{ \pm}$for the initially prepared "magnetic" 

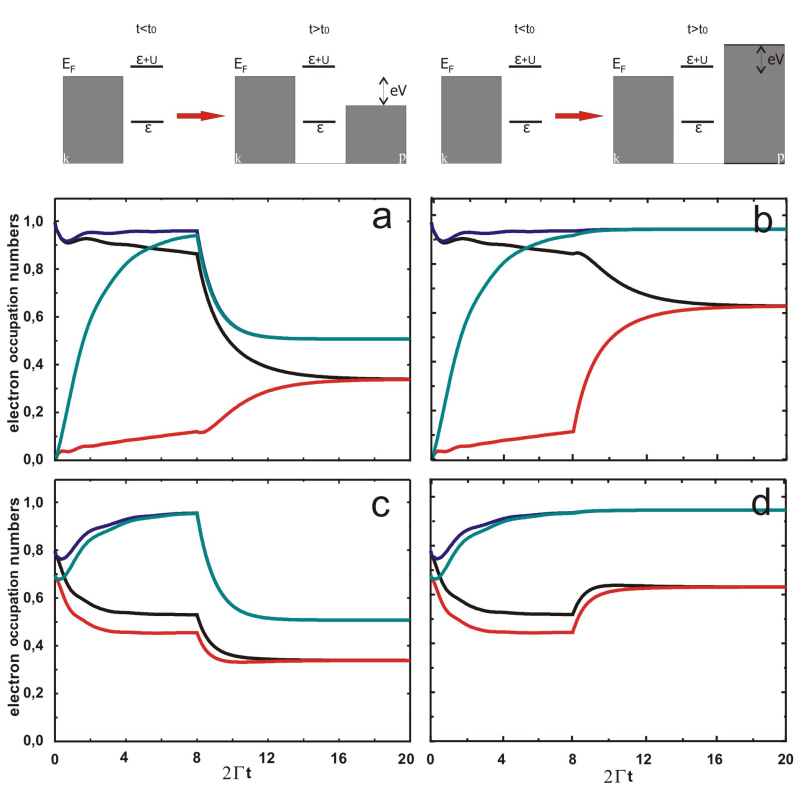

FIG. 2. (Color online) Changing of relaxation regimes of the electron occupation numbers when coupling to the second lead is switched "on" at $2 \Gamma t_{0}=8$ for different initial conditions. Black line demonstrates $n_{1 \sigma}(t)$ and red line $-n_{1-\sigma}(t)$ in the presence of Coulomb correlations. Blue line demonstrates $n_{1 \sigma}(t)$ and green line $-n_{1-\sigma}(t)$ in the absence of Coulomb correlations. a),b) $n_{1 \sigma}=1, n_{1-\sigma}=0$; c),d) $n_{1 \sigma}=0.8$, $n_{1-\sigma}=0.7$. a),c) $\mathrm{eV} / 2 \Gamma=-5.0$ and b),d) $\mathrm{eV} / 2 \Gamma=12.5$. Parameters $U / 2 \Gamma=7.5, \varepsilon / 2 \Gamma=-2.5$, and $\Gamma=1\left(\Gamma_{k}=\Gamma_{p}\right)$ are the same for all the figures.

state for the different constant values of applied bias are depicted in Fig.(3) - Fig.(4). Schemes of the spinpolarized currents directions are shown in Fig.(5). For the large negative values of applied bias voltage (see Fig $3 \mathrm{a}$ and Fig $5 \mathrm{a}$ ) non-stationary spin-polarized currents $I_{p}^{+}(t)$ and $I_{p}^{-}(t)$ are flowing in the same direction in the tunneling contact lead with the Fermi level shifted by the applied bias voltage (lead $p$ ). In this case strong spin polarization of the total current occurs at the initial stage of relaxation as the amplitude of current $I_{p}^{+}(t)$ strongly exceeds the amplitude of current $I_{p}^{-}(t)$. Non-stationary spin-polarized currents $I_{k}^{+}(t)$ and $I_{k}^{-}(t)$ in the lead with $E_{F}=0$ are also flowing in the same direction, but the difference between currents amplitudes is small (see Fig $3 \mathrm{~b}$ and Fig 5 a).

For the positive values of applied bias voltage (see Fig 4 and Fig. 5 p) direction of the non-stationary spin-polarized currents changes to the opposite one in comparison with the case, when large negative bias was applied to the tunneling contact [see Fig $[5$ a]. One can easily distinguish the presence of non-stationary spin-polarized currents $I_{p}^{+}(t)$ and $I_{p}^{-}(t)$ again flowing in the same direction in the tunneling contact lead with the Fermi level shifted by the applied bias voltage (lead $p$ ) (see Fig.4a and Fig 5 b). Non-stationary spin-polarized currents $I_{k}^{+}(t)$ and $I_{k}^{-}(t)$ in the lead with $E_{F}=0$ are also flowing in the same direction, but the difference between currents amplitudes is

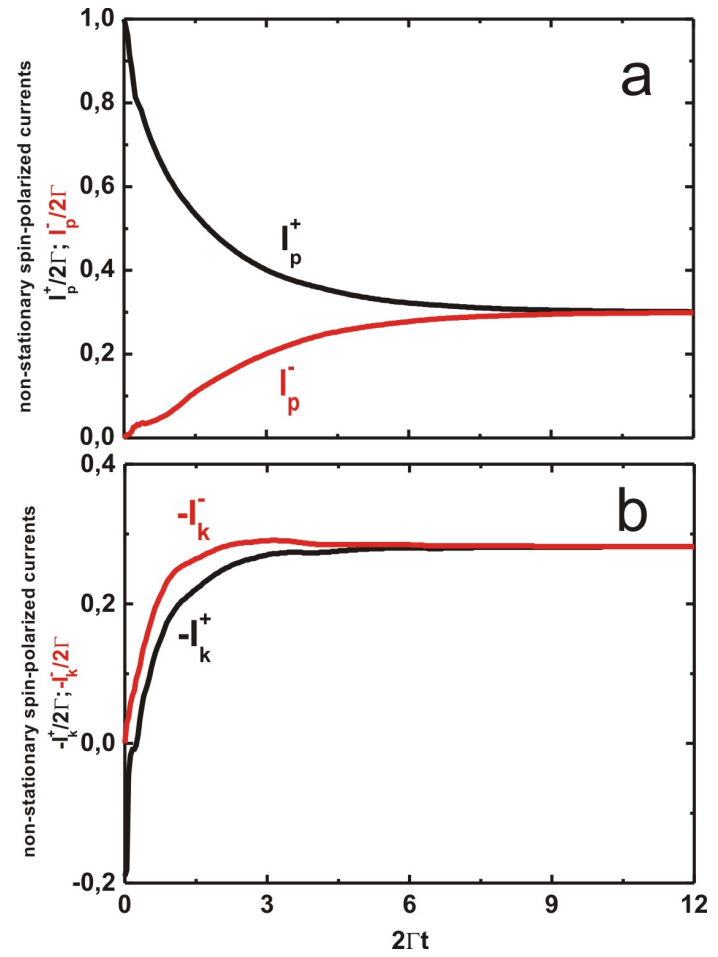

FIG. 3. (Color online) Normalized non-stationary spinpolarized tunneling currents $I_{k(p)}^{+}(t) / 2 \Gamma$ (black line) and $I_{k(p)}^{-}(t) / 2 \Gamma$ (red line). a) Black line $I_{p}^{+}(t)$, red line $I_{p}^{-}(t)$; b) Black line $I_{k}^{+}(t)$, red line $I_{k}^{-}(t)$. Parameters $U / 2 \Gamma=7.5$, $\varepsilon / 2 \Gamma=-2.5, \mathrm{eV} / 2 \Gamma=-5.0$ and $\Gamma=1$ are the same for all the figures. $n_{1 \sigma}(0)=1, n_{1-\sigma}(0)=0$.

quite small (see Fig, 4 b and Fig 5 b). Fig. (33)-(4) demonstrate equal amplitudes of non-stationary spin-polarized currents in the stationary state.

For typical $\Gamma \sim 1 \div 10 \mathrm{meV}$ and $|\varepsilon| \sim 50 \mathrm{meV}^{24,25}$, corresponding to the situation depicted in Fig 3 , Fig 4 the non-stationary spin-polarized current value is about $1 \div 10 \mathrm{nA}\left(1 \mathrm{nA} \simeq 6 \times 10^{9} \mathrm{e} / \mathrm{sec}\right)$.

We revealed, that spin polarization and direction of the non-stationary currents in each lead can be simultaneously inverted by the sudden changing of the applied bias voltage (see Fig 6 and Fig 7). Fig 6a demonstrates that initially spin-polarized non-stationary current with the dominant $I_{p}^{+}(t)$ component changes direction and polarization (component $I_{p}^{-}(t)$ starts to prevail), when the applied bias changes the value from the large negative to the large positive one (system energy scheme changes from the one shown in Fig $5 \mathrm{a}$ to the one demonstrated in Fig 5b). Tunneling current in the another contact lead also changes polarization and direction (see Fig 6b), but the difference between the components with different spins is not so well pronounced.

Opposite situation is depicted in Fig.7 In this case applied bias sign changing leads to the situation when initially spin-polarized non-stationary current with the dominant component $I_{p}^{-}(t)$ changes direction and po- 


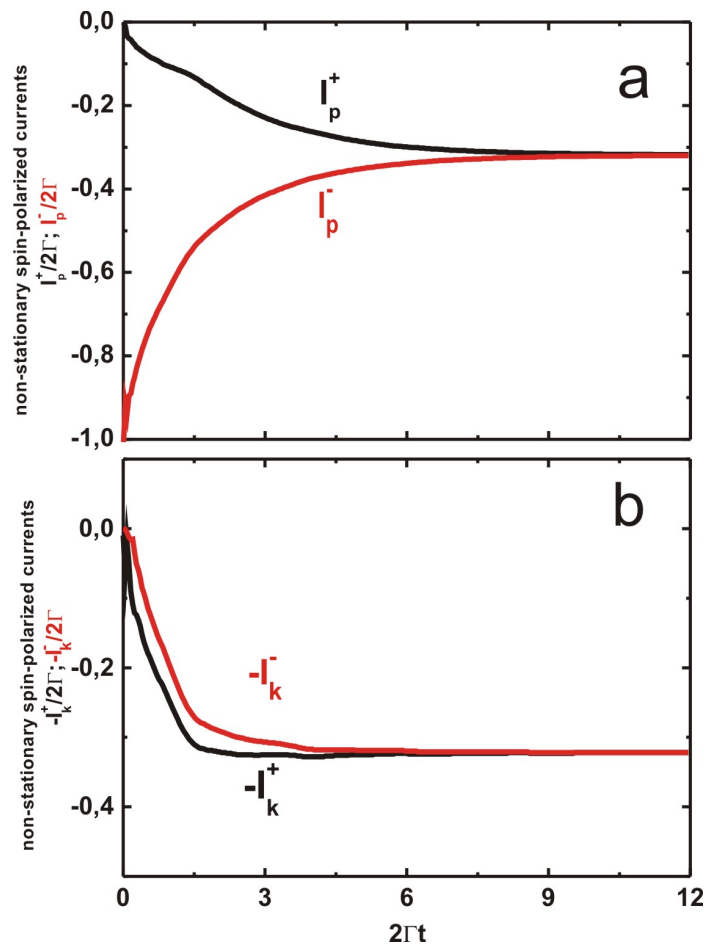

FIG. 4. (Color online) Normalized non-stationary spinpolarized tunneling currents $I_{k(p)}^{+}(t) / 2 \Gamma$ (black line) and $I_{k(p)}^{-}(t) / 2 \Gamma$ (red line). a) Black line $I_{p}^{+}(t)$, red line $I_{p}^{-}(t)$; b) Black line $I_{k}^{+}(t)$, red line $I_{k}^{-}(t)$. Parameters $U / 2 \Gamma=7.5$, $\varepsilon / 2 \Gamma=-2.5, \mathrm{eV} / 2 \Gamma=12.5$ and $\Gamma=1$ are the same for all the figures. $n_{1 \sigma}(0)=1, n_{1-\sigma}(0)=0$.

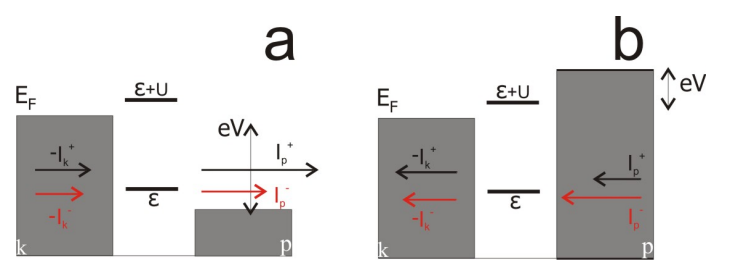

FIG. 5. (Color online) Schemes of the spin-polarized currents directions. Scheme a) corresponds to the results, shown in the Fig, 3 , b) in the Fig 4

larization to the opposite one and component $I_{p}^{+}(t)$ becomes the leading one (see Fig 6a) (system energy scheme changes from the one shown in Fig $5 \mathrm{p}$ to the one demonstrated in Fig 5a).

Corresponding electron occupation numbers behavior is shown in Fig 8, Electron occupation numbers reveal non-monotonic behavior.

\section{STATIONARY CORRELATION FUNCTIONS: FORMALISM AND RESULTS}

The behavior of the local "magnetic" moments can be also analyzed from the time dependence of the stationary correlation functions for the electron occupation num-
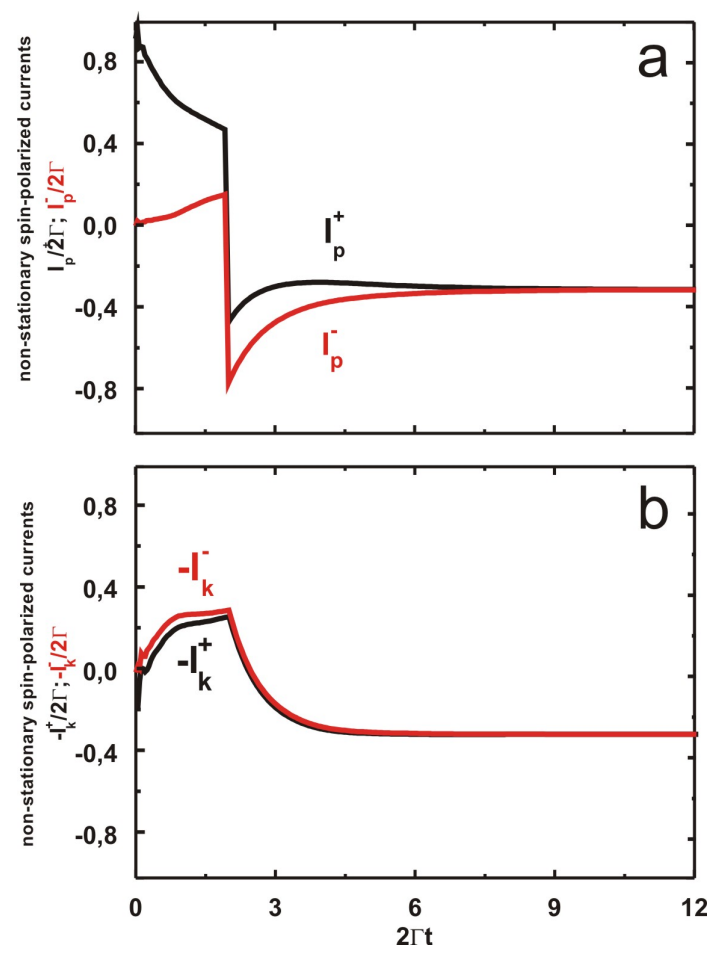

FIG. 6. (Color online) Triggering of the normalized nonstationary spin-polarized tunneling currents in the case, when the value of the applied bias voltage changes at $2 \Gamma t_{0}=2$. a) Black line $I_{p}^{+}(t)$, red line $I_{p}^{-}(t)$; b) Black line $I_{k}^{+}(t)$, red line $I_{k}^{-}(t)$. Parameters $U / 2 \Gamma=7.5, \varepsilon / 2 \Gamma=-2.5, \mathrm{eV} / 2 \Gamma=-7.5$ for $2 \Gamma t<2 \Gamma t_{0}$ and $\mathrm{eV} / 2 \Gamma=7.5$ for $2 \Gamma t>2 \Gamma t_{0}$. Parameter $\Gamma=1$ is the same for all the figures. $n_{1 \sigma}(0)=1, n_{1-\sigma}(0)=0$.

bers:

$$
K^{\sigma \sigma^{\prime}}\left(t-t^{\prime}\right)=<n_{1 \sigma}(t) n_{1 \sigma^{\prime}}\left(t^{\prime}\right)>
$$

Correlation functions $K^{\sigma \sigma^{\prime}}\left(\tau=t-t^{\prime}\right)$ satisfy the system of equations:

$$
\begin{aligned}
& \frac{\partial}{\partial t} K^{+-}=-2 \Gamma \cdot\left[K^{+-}+\Delta N^{T} K^{--}-N_{\varepsilon}^{T} n_{1-\sigma}\right] \\
& \frac{\partial}{\partial t} K^{--}=-2 \Gamma \cdot\left[K^{--}+\Delta N^{T} K^{+-}-N_{\varepsilon}^{T} n_{1-\sigma}\right] .
\end{aligned}
$$

Initial conditions are determined as:

$$
\begin{array}{r}
K^{+-}(t, t)=K^{+-}(0)=\frac{N_{\varepsilon+U}^{T} \cdot N_{\varepsilon}^{T}}{1+\Delta N^{T}}, \\
K^{--}(0)=n_{1}^{s t}=\frac{N_{\varepsilon}^{T}}{1+\Delta N^{T}} .
\end{array}
$$

Time evolution of the correlation functions can be obtained from Eq. 25): 


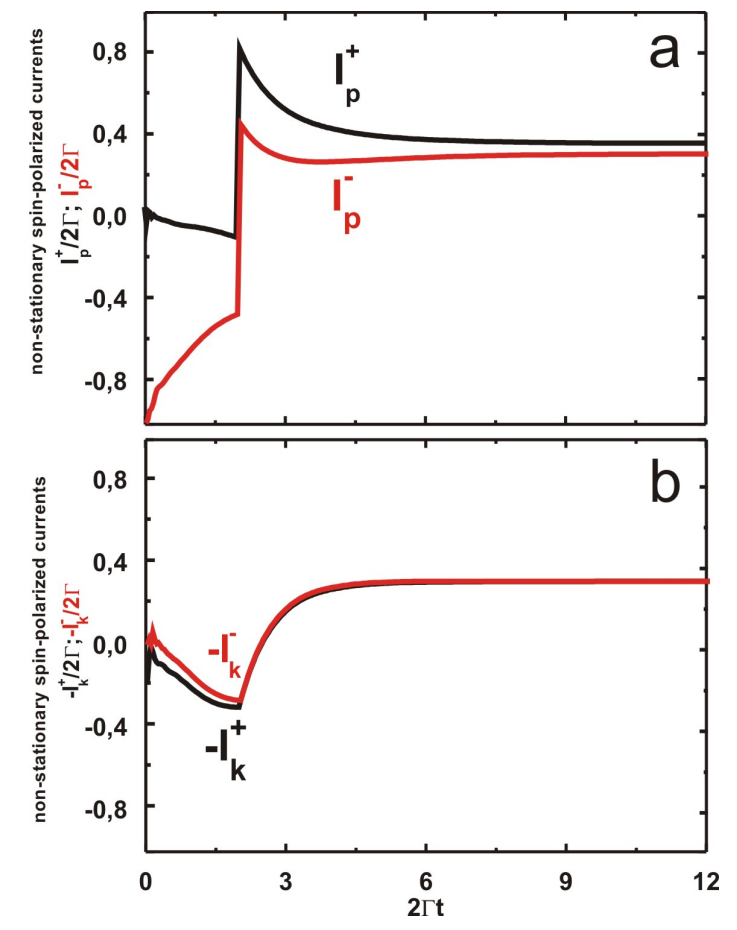

FIG. 7. (Color online) Triggering of the normalized nonstationary spin-polarized tunneling currents in the case, when the value of the applied bias voltage changes at $2 \Gamma t_{0}=2$. a) Black line $I_{p}^{+}(t)$, red line $I_{p}^{-}(t)$; b) Black line $I_{k}^{+}(t)$, red line $I_{k}^{-}(t)$. Parameters $U / 2 \Gamma=7.5, \varepsilon / 2 \Gamma=-2.5, \mathrm{eV} / 2 \Gamma=7.5$ for $2 \Gamma t<2 \Gamma t_{0}$ and $e V / 2 \Gamma=-7.5$ for $2 \Gamma t>2 \Gamma t_{0}$. Parameter $\Gamma=1$ is the same for all the figures. $n_{1 \sigma}(0)=1, n_{1-\sigma}(0)=0$.

$$
\begin{gathered}
K^{+-}\left(\tau=t-t^{\prime}\right)=\frac{\left(N_{\varepsilon}^{T}\right)^{2}}{\left(1+\Delta N^{T}\right)^{2}}\left(1-e^{\lambda_{2}^{T} \tau}\right)+ \\
+\frac{N_{\varepsilon}^{T}\left(N_{\varepsilon+U}^{T}-1\right)}{2\left(1+\Delta N^{T}\right)} e^{\lambda_{1}^{T} \tau}+\frac{N_{\varepsilon}^{T}\left(N_{k \varepsilon+U}^{T}+1\right)}{2\left(1+\Delta N^{T}\right)} e^{\lambda_{2}^{T} \tau}, \\
K^{--}\left(\tau=t-t^{\prime}\right)=\frac{\left(N_{\varepsilon}^{T}\right)^{2}}{\left(1+\Delta N^{T}\right)^{2}}\left(1-e^{\lambda_{2}^{T} \tau}\right)+ \\
+\frac{N_{\varepsilon}^{T}\left(N_{\varepsilon+U}^{T}+1\right)}{2\left(1+\Delta N^{T}\right)} e^{\lambda_{2}^{T} \tau}+\frac{N_{\varepsilon}^{T}\left(1-N_{\varepsilon+U}^{T}\right)}{2\left(1+\Delta N^{T}\right)} e^{\lambda_{1}^{T} \tau} .
\end{gathered}
$$

The behavior of the stationary correlation functions for the localized electrons occupation numbers with the different spin orientation is depicted in Fig.9. It is clearly evident, that for the deep energy levels correlation functions time evolution is much slower, than for the states with the shallow energy levels.

For $\tau \rightarrow \infty$ correlation functions turn to the product of the decoupled electronic occupation numbers mean values:

$$
K^{+-s t}=K^{--s t} \simeq\left(\frac{N_{\varepsilon}^{T}}{1+\Delta N^{T}}\right)^{2}
$$

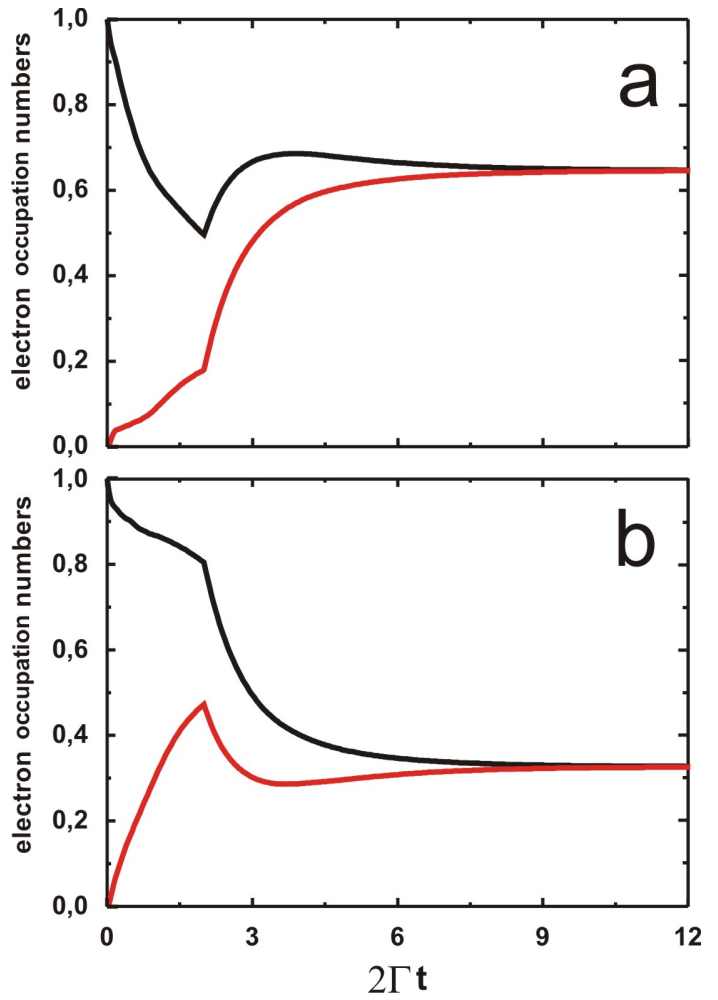

FIG. 8. (Color online) Electron occupation numbers time evolution in the case, when the value of applied bias voltage changes at $2 \Gamma t_{0}=2$. Black line $n_{1 \sigma}(t)$, red line $n_{1-\sigma}(t)$; a). $e V / 2 \Gamma=-7.5$ for $t<t_{0}$ and $e V / 2 \Gamma=7.5$ for $\left.2 \Gamma t>2 \Gamma t_{0} \mathrm{~b}\right)$. $e V / 2 \Gamma=7.5$ for $t<t_{0}$ and $e V / 2 \Gamma=-7.5$ for $2 \Gamma t>2 \Gamma t_{0}$. Parameters $U / 2 \Gamma=7.5, \varepsilon / 2 \Gamma=-2.5$ and $\Gamma=1$ are the same for all the figures. $n_{1 \sigma}(0)=1, n_{1-\sigma}(0)=0$.

So for $t<\frac{1}{\left|\lambda_{1}^{T}\right|}$ the "magnetic" correlations are still present in the system.

\section{CONCLUSION}

We analyzed time evolution of the opposite spin electron occupation for the single-localized state with the Coulomb interaction coupled to two reservoirs in the presence of applied bias voltage. We revealed that in the presence of the second reservoir with non-zero applied bias, "magnetic" state can be distinguished from the "paramagnetic" one by analyzing time evolution of the electron occupation numbers. Typical time scales strongly depend on the value of applied bias and initial conditions.

We revealed that non-stationary spin-polarized currents can flow in the both leads and their direction and polarization depend on the value of applied bias. We revealed, that spin polarization and the direction of the non-stationary currents in each lead can be simultaneously inverted by the sudden changing of the applied bias voltage. But in the stationary state occupation numbers for the electrons with the opposite spins have the same 

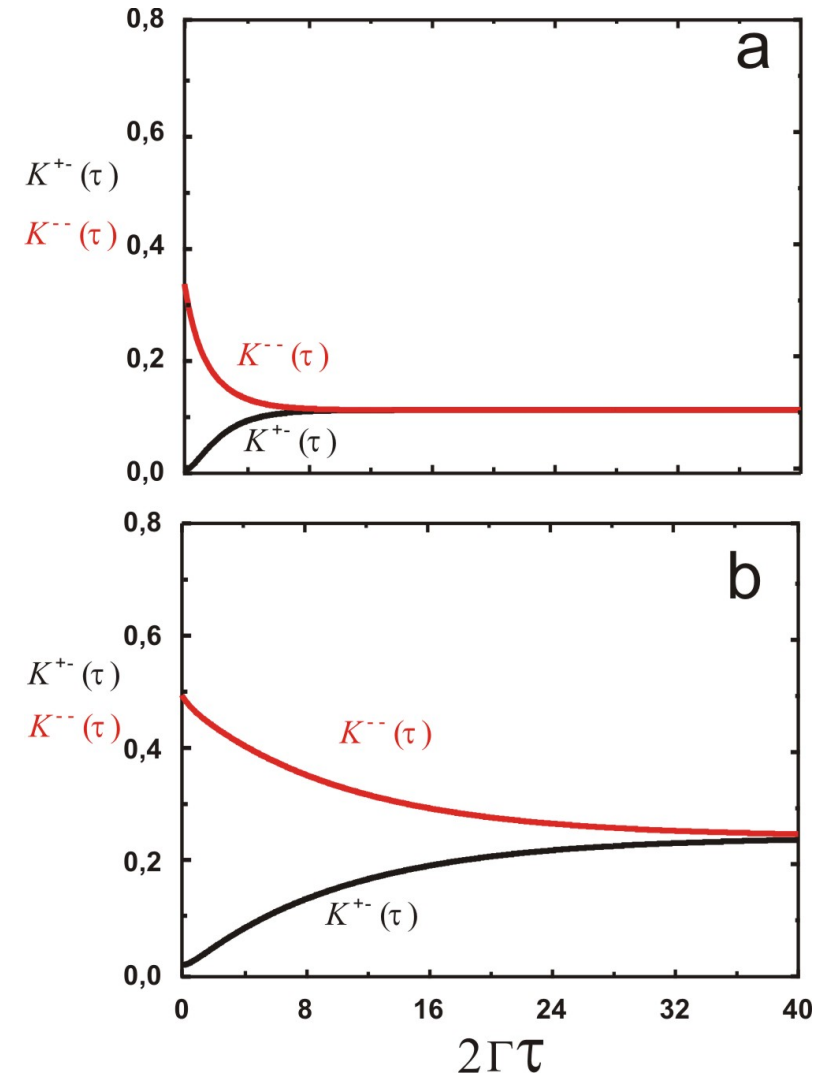

FIG. 9. (Color online) Correlation functions time evolution for the different initial conditions. Black lines demonstrate $K_{\tau}^{+-}$, red line $-K_{\tau}^{--}$. a) $\mathrm{eV} / 2 \Gamma=-5$; b) $\mathrm{eV} / 2 \Gamma=2.5$. parameters $U / 2 \Gamma=7.5, \varepsilon / 2 \Gamma=-2.5$ and $\Gamma=1$ are the same for all the figures. values. Spin polarized tunneling currents in each lead also become equal.
We also investigated the changes of the time evolution regimes when the second lead is switched on at the particular time moment. We found out that switching on of the second lead with the non-zero applied bias destroys long-living "magnetic" moment.
This work was supported by RFBR grant $16-32-$ $60024 \mathrm{~mol}-a-d k$ and by RFBR grant $14-02-00434$.
* vmantsev@gmail.com

1 Semiconductor Spintronics and Quantum Computation, edited by D.D. Awschalom, D. Loss, N. Samarth, Nanoscience and Technology (Springer, Berlin, 2002).

2 E.Y. Tsymbal, O. Mryasov, P.R. LeClair, J. Phys.: Condens. Matter 15, R109, (2003)

3 I. Žutić, J. Fabian, S. Das Sarma, Rev. Mod. Phys. 76, 323, (2004)

${ }^{4}$ H.J. Zhu, M. Ramsteiner, H. Kostial, M. Wassermeier, H.P. Schonherr, K.H. Ploog, Phys. Rev. Lett. 87, 116601, (2001)

5 Y. Ohno, D.K. Young, B. Beschoten, F. Matsukura, H. Ohno, D.D. Awschalom, Nature(London) 402, 790, (1999)

${ }^{6}$ R. Fiederling, M. Keim, G. Reuscher, W. Ossau, G. Schmidt, A. Waag, L.W. Molenkamp, Nature(London) 402, 787, (1999)

7 H.B. Heersche, Th. Schapers, J. Nitta, H. Takayanagi, Phys. Rev. B 64, 161307, (2001)

8 J.C. Egues, Phys. Rev. Lett. 80, 4578, (1998)

9 V.I. Perel', S.A. Tarasenko, I.N. Yassievich, S.D. Ganichev, V.V. Bel'kov, W. Prettl, Phys. Rev. B 67, 201304, (2003)

10 M.M. Glazov, P.S. Alekseev, M.A. Odnoblyudov, V.M. Chistyakov, S.A. Tarasenko, I.N. Yassievich, Phys. Rev. $B$ 71, 155313, (2005)
11 T. Koga, J. Nitta, H. Takayanagi, S. Datta, Phys. Rev. Lett. 88, 126601, (2002)

12 A. Voskoboynikov, S.S. Liu, C.P. Lee, Phys. Rev. B 58, 15397, (1998)

13 I. Bar-Joseph, S.A. Gurvitz, Phys.Rev B, 44, 3332, (1991).

14 S.A. Gurvitz, M.S. Marinov, Phys.Rev A, 40, 2166, (1989).

15 P.I. Arseyev, N.S. Maslova, V.N. Mantsevich, European Physical Journal B, 85(7), 249, (2012).

16 C.A. Stafford, N.S. Wingreen, Phys. Rev. Lett., 76, 1916 , (1996).

17 B.L. Hazelzet, M.R. Wegewijs, T. H. Stoof, Y.V. Nazarov, Phys. Rev. B, 63, 165313, (2001).

18 E. Cota, R. Aguado, G. Platero, Phys. Rev. Lett., 94, 107202, (2005).

19 P.I. Arseyev, N.S. Maslova, V.N. Mantsevich, Solid State Comm., 152, 1545, (2012).

20 V.N. Mantsevich, N.S. Maslova, P.I. Arseyev, JETP, 118(1), 136, (2014).

21 L.D. Contreras-Pulido, J. Splettstoesser, M. Governale, J. Konig, M. Buttiker, Phys. Rev. B, 85, 075301, (2012).

22 Florian Elste, David R. Reichman, and Andrew J. Millis, Phys. Rev. B, 81, 205413, (2010).

23 D. M. Kennes, S. G. Jakobs, C. Karrasch, V. Meden, Phys. Rev. B, 85, 085113, (2012). 
${ }^{24}$ S. Amaha, W. Izumida, T. Hatano, S. Teraoka, S. Tarucha, J. A. Gupta, and D. G. Austing, Phys. Rev. Lett., 110,
(2013), 016803.

25 J. Fransson Phys. Rev. B, 69, 201304, (2004). 